\title{
A New Class of Time-Consistent Dynamic Risk Measures and its Application
}

\author{
Rui Gao, Zhiping Chen \\ School of Mathematics and statistics, Xi'an Jiaotong University, Xi'an, China \\ Email:rgao.xjtu@hotmail.com, zchen@mail.xjtu.edu.cn
}

Received 2012

\begin{abstract}
We construct a new time consistent dynamic convex cash-subadditive risk measure in this paper. Different from existing measures, both potential loss and volatility of risky objects are considered. Based on a one-period measure that distorts financial values, punishes downside risk yet rewards upside potential, a dynamic time consistent version is constructed recursively through a modified translation property. We then establish a portfolio selection model and give its optimal condition.
\end{abstract}

Keywords: Dynamic Risk Measure; Time-Consistent; Cash-Subadditive; Portfolio Optimization; Stochastic Programming

\section{Introduction}

Financial activity is teemed with risk, therefore it is crucial to construct reasonable risk measures and utilize them on the optimal portfolio selection. One popular definition of risk is volatility of random return of portfolio, originated from Markowitz's prominent mean-variance model. Following him, hundreds of moment-based risk measures were proposed, such as Mean Absolute Deviation [1] and Lower Partial Moment [2]. Another common notion of risk is potential downside loss below a certain target. Correspondingly, a number of downside risk measures has been suggested in the literature, such widely used measures as Value-at-Risk $(\mathrm{VaR})$ and Conditional Value-at-Risk (CVaR). In all the above financial risk measures, the attention is put on either the volatility of random return or the potential loss. Nevertheless, this is insufficient. Both these two aspects should be considered simultaneously. Only concentrating on the volatility of return ignores the information on the degree of potential loss; while merely emphasizing potential loss not only neglects the dispersion of future return but also throws away upside data. What's more, when considering the volatility of random return, many researchers punish both downside risk and upside potential. However, the volatility of random return above certain target implies the potential of gaining much more than expected. A higher upside variability generally indicates a higher possibility to acquire good upside performance, which is desirable for each rational investor. Hence, upside potential should be rewarded. Based on the analysis above, we believe that when defining risk and its measure, we should consider both the potential loss and volatility into consideration and distinguish between downside risk and upside potential.

Generally speaking, an ideal risk measure should satisfy some properties. In their seminal paper [3], Artzner, Delbaen, Eber, Heath established an axiomatic notion of coherent risk measures. They proposed that an ideal risk measure should satisfy four properties: monotonicity, subadditivity, positive homogeneity and translation-invariance. Though it has been accepted by many scholars, it is not perfect. For example, positive homogeneity sometimes does not hold because a financial position's risk increases in a nonlinear way with its volume due to liquidity risk. Hence, Follmer and Schied [4] replaced subadditivity and positive homogeneity by convexity and established a more general concept of convex risk measures. In addition, translation-invariance is questioned in [5] since the ambiguity on interest rates and is suggested to be replaced with cash-subadditivity, which implies that additional loss of some amount of money is covered by an additional reserve of the same amount. Hence, we believe that for one-period risk measures it is reasonable to assume monotonicity, convexity and cash-subadditivity. From the perspective of economics and finance, an ideal risk measure should reflect investor's risk-averse attitude because risk is always a subjective notion [6].

During the recent decade, dynamic risk measure has attracted many researchers and practitioners, of which the most important feature is time consistency describing how risk assessments at different times are interrelated. 
By certain translation property, which corresponds to translation-invariance in one-period setting, time consistent risk measures can be completely defined by conditional risk measures recursively [7]. Thus, an usual way to construct time-consistent risk measures is establishing a static risk measure first and extending it to dynamic setting by translation property. As illustrated later, existed translation property cannot reflect risk aversion and we will modified it into another version.

Bearing in mind the above limitation in existed risk measures, a new class of time consistent dynamic cash-subadditive convex risk measure is constructed. Comparing with existing measures, our new risk measure has the following advantages: we take into account the potential loss and volatility of both downside risk and upside potential, and thus the whole domain distribution is utilized, which makes the new measure superior for finding robust and stable investment decisions; by suitably selecting the parameters in the model, our risk measure can explicitly reflect the investor's risk attitude; when the risk measure is applied to portfolio selection model, we give its optimality condition, which is useful in determining the stochastic dual dynamic programming method to solve the risk-averse multistage problem.

This paper is organized as follows. Section 2.1 gives the definition and property of the new one-period risk measure, which is then extended to dynamic setting in Section 2.2. We apply the risk measure to portfolio selection model in Section 3 and presents our conclusion in Section 4.

\section{The New Risk Measure and its Properties}

\subsection{One-Period Setting}

We first consider a one-period framework. Given a probability space $(\Omega, F, P)$, denote the random cost, discounted by certain numéraire, of at time $T$ by essentially bounded random variable $X$ in $L^{\infty}(\mathrm{F})$. A one-period risk measure is a mapping $\rho: L^{\infty}(\mathrm{F}) \rightarrow \square$. A larger value of $\rho$ implies a riskier $\operatorname{cost} X$. For $a \in \square$, we denote $[a]^{+}$by $\max \{0, a\}$. The notation ":=" means "equal by definition". For a random variable $X, \mathrm{E}[X]$ denotes its expectation; $x_{\alpha}$ is the $\alpha$ quantile of $X$. We denote the indicator function of set $A$ by $\mathbf{1}_{A}$.

For reasons demonstrated in the introduction, we propose here a new type of risk measure that takes into account the potential loss, downside risk, upside potential and risk aversion. In order to illustrate the derivation of our new risk measure, we look back on the notable downside risk measure $\mathrm{VaR}$, which is defined as $\operatorname{VaR}_{\alpha}(X):=x_{1-\alpha}$. When the random cost $X$ is reduced by $x_{1-\alpha}$ amount of money, it becomes acceptable in the sense that the random cost $X-x_{\alpha}$ is less than zero up to a loss with probability $\alpha$. However, as pointed out before, the volatility of such random cost should also be meas- ured. We use absolute deviation to measure the upside potential and downside risk separately. The average downside deviation is $\mathrm{E}\left[X-x_{1-\alpha} \mid X \geq x_{1-\alpha}\right]$, which equals to $\alpha^{-1} \mathrm{E}\left[X-x_{1-\alpha}\right]^{+}$. Similarly, the upside potential is $\left(1-\alpha^{-1}\right) \mathrm{E}\left[x_{1-\alpha}-X\right]^{+}$. Since we punish downside risk and reward upside potential, the volatility of $X-x_{1-\alpha}$ is $\lambda \alpha^{-1} \mathrm{E}\left[X-x_{1-\alpha}\right]^{+}-(1-\lambda)(1-\alpha)^{-1} \mathrm{E}\left[x_{1-\alpha}-X\right]^{+}$, where $\lambda$ reflects the asymmetry between downside and upside. To combine the potential loss and volatility together, a simple way is by linear weight. It is not difficult to show that to make the weighted sum be monotone, they have to have equal weight. Moreover, taking into risk aversion into consideration, all the financial values aforementioned, including $X$ and $X-x_{1-\alpha}$, should be distorted by a monotonically decreasing convex function $w(\cdot)$. Such property of $w$ indicates that risk-averse investors emphasize more on undesirable situation. For normalization condition, we require $w(0)=0$. Finally, we define our new risk measure as follows.

Definition 1. Given $\lambda \in[0,1], \alpha \in(0,1)$, the new one-period risk measure $\rho_{\lambda, \alpha}: L^{\infty}(\mathrm{F}) \rightarrow \square$ is defined as

$$
\begin{aligned}
\rho_{\lambda, \alpha}(X) & =w\left(x_{1-\alpha}\right)+\lambda \alpha^{-1} \mathrm{E}\left[w(X)-w\left(x_{1-\alpha}\right)\right]^{+} \\
& -(1-\lambda)(1-\alpha)^{-1} \mathrm{E}\left[w\left(x_{\alpha}\right)-w(X)\right]^{+}
\end{aligned}
$$

where $x_{\alpha}=\inf \{x \in \square: P[X \leq x] \geq \alpha\}$ and $w(\cdot)$ is a monotonically increasing convex continuous function satisfying normalized condition $w(0)=0$.

Rewriting the expectation in Equation (1) in integral form, the measure has an equivalent form which facilitates us to study its property, demonstrating by the following proposition:

Proposition 1. For any $0<\alpha \leq \lambda \leq 1$, the risk measure $\rho_{\lambda, \alpha}$ defined by (1) can be equivalently written as

$$
\rho_{\lambda, \alpha}(X)=\frac{1-\lambda}{1-\alpha} \mathrm{E}[w(X)]+\frac{\alpha-\lambda}{\alpha(1-\alpha)} \int_{1-\alpha}^{1} w\left(x_{u}\right) d u
$$

Obviously, if $\lambda=1, \quad \rho_{1, \alpha}(X)=\int_{1-\alpha}^{1} w\left(x_{u}\right) d u$ is $\alpha$ time of $\operatorname{TNT}_{\alpha}(X)$ defined in [8]; if $w(x)=x$, $\rho_{\lambda, \alpha}(X)=(1-\beta) \mathrm{E}[X]+\beta \operatorname{CVaR}_{\alpha}(X) \quad, \quad$ where $\beta=(1-\lambda)(1-\alpha)^{-1}$, is appeared in [9]; further if $\alpha=1 / 2$, $\left.\rho_{\lambda, 1 / 2}=\mathrm{E}[X]+2 \lambda \mathrm{E}\left[X-x_{1 / 2}\right]^{+}-21-\lambda\right) \mathrm{E}\left[x_{1 / 2}-X\right]^{+} \quad$ is the deviation measure suggested in [10]. Therefore, our new risk measure can be regarded as extensions to all these risk measures.

The choice of depends on the investor's attitude toward risk controls the heavy tails of loss distribution. Typical decreasing convex functions are $\beta_{1}[X]^{+}-\beta_{2}[-X]^{+}$ 
$\left(\beta_{1}>\beta_{2}>0\right), \exp (\beta x)-1(\beta>0), x^{\beta}(\beta \geq 1)$. As for concrete selection of and corresponding parameters, one can refer to [8] for a detailed discussion.

The following proposition shows that under certain mild specification, the new risk measure satisfies several desirable mathematical properties.

Proposition 2. For any $0<\alpha \leq \lambda \leq 1$ and $w(\cdot)$ that is differentiable and satisfies $0 \leq w^{\prime} \leq 1$, then the risk measure defined in Equation (1) is a law-invariant, convex, cash-subadditive risk measure.

Proof. The first two are direct corollaries of Theorem 1. To prove the cash-subadditivity, we show that $\rho_{\lambda, \alpha}(X+m) \leq \rho_{\lambda, \alpha}(X)+m$. Rewriting the new risk measure as $\quad \rho_{\lambda, \alpha}=\int_{0}^{1} w\left(x_{u}\right) \phi(u) d u \quad$, where $\phi(u):=\lambda / \alpha \mathbf{1}_{[0, \alpha)}(u)+(\lambda-\alpha) /(1-\alpha) \mathbf{1}_{[\alpha, 1]}(u) \quad, \quad$ by $(x+m)_{u}=x_{u}+m$, we have

$$
\begin{aligned}
\rho_{\lambda, \alpha}(X+m) & =\int_{0}^{1} w\left((x+m)_{u}\right) \phi(u) d u \\
& =\int_{0}^{1} w\left(x_{u}+m\right) \phi(u) d u \\
& =\int_{0}^{1}\left[w\left(x_{u}\right)+w^{\prime}\left(\xi_{u}\right) m\right] \phi(u) d u \\
& \leq \int_{0}^{1} w\left(x_{u}\right) \phi(u) d u+m \\
& =\rho_{\lambda, \alpha}(X)+m,
\end{aligned}
$$

where $\xi_{u} \in\left[x_{u}-m, x_{u}\right]$ is determined by the mean value theorem.

Besides, Equation (1) contains two parameters $\lambda$ and $\alpha$, which can be flexibly reflect investor's attitude toward risk. $\lambda$ is a factor linearly adjusting the balance between downside risk and upside potential; is the confidence level that the investors can accept. More specifically, we have the following theorem.

Proposition 3. The coherent risk measure $\rho_{\lambda, \alpha}$ is increasing with respect to $\lambda$, decreasing with respect to $\alpha$, and continuous with respect to $\alpha$ and $\lambda$.

The monotonicity property of $\rho_{\lambda, \alpha}$ with respect to $\lambda / \alpha$ can be used to reflect the investor's attitude toward risk. Concretely, the increasing property of $\rho_{\lambda, \alpha}$ with respect to $\lambda$ indicate that the greater the $\lambda$, the larger the $\rho_{\lambda, \alpha}$. Investors who adopt a larger $\lambda$ treat $X$ riskier than those who choose a smaller $\lambda$. They have a stronger tendency to risk aversion because the concentrate more on downside risk than on upside potential. When $\lambda=1$, investors only consider downside risk. On the other hand, the decreasing property of $\rho_{\lambda, \alpha}$ with respect to $\alpha$ means that $\rho_{\lambda, \alpha}$ with large $\alpha$ should be connected with the less risk-averse investor.
They are less conservative in the sense that they bear a larger probability of loss.

Stochastic dominance rules are often utilized to judge a new risk measure. From the point of view of utility theory, it is desirable for a risk measure to preserve second order stochastic dominance (SSD). An equivalent definition of SSD in terms of quantile function is the following: $X \succ_{S S D} Y$ if and only if

$$
\int_{0}^{p}\left(x_{u}-y_{u}\right) d u \leq 0, \forall 0 \leq p \leq 1,
$$

and there is a strict inequality of at least one $p_{0}$.

Proposition 4. The risk measure $\rho_{\lambda, \alpha}$ preserves second order stochastic dominance.

Proof. It suffices to prove the case when two random variables $X \succ_{S S D} Y$ whose quantile functions satisfy $x_{u} \not \equiv y_{u}$ in any interval $(a, b) \subset[0,1]$. Since the quantile function is right-continuous, there exists only countable intersection of $x_{u}$ and $y_{u}$. If there is no intersection, it follows that $x_{u} \leq y_{u}$ and thus $\rho_{\lambda, \alpha}(X) \leq \rho_{\lambda, \alpha}(Y)$ due to the monotonicity of $w(\cdot)$. Otherwise denote all the intersection point of $x_{u}$ and $y_{u}$ by $\left\{u_{n}\right\}_{n=1}^{N}, 1 \leq N \leq \infty$, and $u_{0}:=0, u_{N+k}:=1,\left(k \in \square^{+}\right)$. Then we have

$$
\begin{aligned}
& \rho_{\lambda, \alpha}(X)-\rho_{\lambda, \alpha}(Y)=\sum_{n=0}^{N} \int_{u_{n}}^{u_{n+1}}\left[w\left(x_{u}\right)-w\left(y_{u}\right)\right] \phi(u) d u \\
& =\sum_{n=0}^{[N / 2]} \int_{u_{2 n}}^{u_{2 n+1}}\left[w\left(x_{u}\right)-w\left(y_{u}\right)\right] \phi(u) d u \\
& -\int_{u_{2 n+1}}^{u_{2 n+2}}\left[w\left(y_{u}\right)-w\left(x_{u}\right)\right] \phi(u) d u \\
& =\sum_{n=0}^{[N / 2]} w^{\prime}\left(\xi_{n}\right) \int_{u_{2 n}}^{u_{2 n+1}}\left(x_{u}-y_{u}\right) \phi(u) d u \\
& -w^{\prime}\left(\eta_{n}\right) \int_{u_{2 n+1}}^{u_{2 n+2}}\left(y_{u}-x_{u}\right) \phi(u) d u \\
& \leq 0
\end{aligned}
$$

where $\xi_{n} \in\left[u_{2 n}, u_{2 n+1}\right] \quad$ and $\quad \eta_{n} \in\left[u_{2 n+1}, u_{2 n+2}\right] \quad$ are determined by the mean value theorem. The last inequality is deduced by the convexity of $w(\cdot)$ and the monotonicity of $\phi(u)$.

At the end of this section, we consider the computation and minimization of $\rho_{\lambda, \alpha}$. Let $X:=g(x, \omega)$ be the random cost associated with the decision vector $x \in \mathrm{G}$, representing element of feasible set $G$, and the random vector $\omega$, standing for the uncertainties in the market which affects the random cost, such as capital gains and dividends. Similar to the arguments in [11], we have the following proposition.

Proposition 5. Introducing the following auxiliary function 
$G_{\lambda, \alpha}(x, \eta)=\frac{1-\lambda}{1-\alpha} E[w(g(x, \omega))]+\frac{\lambda-\alpha}{1-\alpha}\left\{\eta+\alpha^{-1} E\left[(w(g(x, \omega))-\eta)^{+}\right]\right\}$, then $\rho_{\lambda, \alpha}$ has an equivalent form

$$
\rho_{\lambda, \alpha}(x)=\min _{\eta \in \square} G_{\lambda, \alpha}(x, \eta) .
$$

Minimization of $\rho_{\lambda, \alpha}$ with respect to $X \in \mathrm{X}$ is equivalent to

$$
\min _{x \in \mathrm{G}} \rho_{\lambda, \alpha}(x)=\min _{(x, \eta) \in \mathrm{G} \times \square} G_{\lambda, \alpha}(x, \eta) .
$$

Moreover, we have

$$
\begin{aligned}
\left(x^{*}, \xi^{*}\right) & \in \underset{(x, \eta) \in G \times \square}{\arg \min } \Leftrightarrow \\
x^{*} & \in \underset{x \in \mathrm{G}}{\arg \min } \rho_{\lambda, \alpha}(x), \eta^{*} \in \underset{\eta \in \square}{\arg \min } G_{\lambda, \alpha}(x, \eta) .
\end{aligned}
$$

\subsection{Multi-period setting}

In this section we extend our new one-period risk measure $\rho_{\lambda, \alpha}$ to the dynamic setting. Consider a filtered probability space $\left(\Omega, \mathrm{F},\left(\mathrm{F}_{t}\right)_{t=1}^{T}, \mathrm{P}\right)$, where $\mathrm{F}_{1}=\{\varnothing, \Omega\}$ and $\mathrm{F}_{T}=\Omega$, and an adapted stochastic process $X_{t}, t=1, \ldots, T$, representing discounted random return process. Define the space $\mathrm{L}_{t}=\mathrm{L}^{\infty}\left(\Omega, \mathrm{F}_{t}, \mathrm{P}\right)$ and $\mathrm{L}_{t, T}=\mathrm{L}_{t} \times \cdots \mathrm{L}_{T}$. The one-period risk measure naturally induces a sequence of one-period conditional risk measure $\left\{\rho_{t}\right\}_{t=1}^{T-1}: \mathrm{L}_{t} \rightarrow \square:$

$$
\begin{aligned}
\rho_{t}(X) & =\frac{1-\lambda_{t}}{1-\alpha_{t}} \mathrm{E}\left[w(X) \mid \mathrm{F}_{t}\right] \\
& +\frac{\lambda_{t}-\alpha_{t}}{\alpha_{t}} \inf _{\eta \in \square}\left\{\eta+\alpha_{t}^{-1} \mathrm{E}\left[(w(X)-\eta)^{+} \mid \mathrm{F}_{t}\right]\right\},
\end{aligned}
$$

A dynamic risk measure for stochastic process $X_{t}, \ldots, X_{T}$ is a sequence of conditional risk measures $\left\{\rho_{t, T}\right\}_{t=1}^{T-1}$, where $\rho_{t, T}: \mathrm{L}_{t, T} \rightarrow \mathrm{L}_{t}$ assesses the risk of the sequence $X_{t}, \ldots, X_{T}$ from the perspective of time $t$. Our aim is to construct the dynamic risk measure $\left\{\rho_{t, T}\right\}_{t=1}^{T-1}$ based on the conditional risk measure $\left\{\rho_{t}\right\}_{t=1}^{T-1}$.

The key to constructing dynamic risk measures from one-period ones is the translation property, which arises from translation-invariance property of one-period risk measure. A static risk measure $\rho$ satisfies translation-invariance property if for all $m \in \square$, $\rho(X+m)=\rho(X)+m$, which implies cash-invariance, i.e., $\rho(m)=m$. This suggests the risk of a riskless cost, in terms of potential loss, can be described as the its present value. Then the corresponding translation property in existed papers is for all $\left\{X_{t}\right\} \in \mathrm{L}_{1, T}$ and $t=1, \ldots, T-1$,

$$
\rho_{t, T}\left(X_{t}, X_{t+1}, \ldots, X_{T}\right)=X_{t}+\rho_{t, T}\left(0, X_{t+1}, \ldots, X_{T}\right)
$$

Condition (3) indicates that the risk of a stochastic process $\left(X_{t}, \ldots, X_{T}\right)$ from the perspective of time $t$ is the aggregation of its riskless component $X_{t}$ and its risky component $\left(X_{t+1}, \ldots, X_{T}\right)$, and by translation-invariance property, the risk of the riskless component $X_{t}$ is its opposite of its value. Nevertheless, when taking into account the interest rate [5] and more importantly, the investor's risk-averse behavior, it is better to measure riskless object's risk by a distortion of its value instead of itself, i.e., the cash-invariance and corresponding translation-invariance should be replaced with

$$
\rho(m)=w(m), \rho(X+m)=\rho(X)+w(m), m \in \square
$$

where $w(\cdot)$ is a monotonically decreasing convex continuous function. The monotonicity of $w(\cdot)$ guarantees that the smaller the $m$, the riskier it is, and the convexity entails risk-aversion. Intuitively speaking, the dynamic risk measure $\left\{\rho_{t, T}\right\}_{t=1}^{T-1}$ induced by conditional risk measure sequence $\left\{\rho_{t}\right\}_{t=1}^{T-1}$ should satisfies

$$
\rho_{t, t+1}\left(0, X_{t+1}\right)=\rho_{t}\left(X_{t+1}\right), t=1, \ldots, T-1,
$$

which identifies the conditional risk measure $\rho_{t}$ with the dynamic risk measure for two-period process $\rho_{t, t+1}$.

According to (4) and (5), translation property (3) is modified into for all $\left\{X_{t}\right\} \in \mathrm{L}_{1, T}$,

$\rho_{t, T}\left(X_{t}, X_{t+1}, \ldots, X_{T}\right)=w_{t}\left(X_{t}\right)+\rho_{t}\left(\rho_{t+1, T}\left(X_{t+1}, \ldots, X_{T}\right)\right)$,

where $w_{t}(\cdot)$ is a monotonically decreasing convex continuous function.

Based on the above analysis, we define our new dynamic risk measure as follows.

Definition 3. Let $\left\{w_{t}\right\}_{t=1}^{T}$ is a sequence of monotonically decreasing convex differentiable function satisfying for all $1 \leq t \leq T, w_{t}(0)=0$ and $-1 \leq w^{\prime}(t) \leq 0$. The new time consistent dynamic risk measure $\left\{\rho_{t, T}\right\}_{t=1}^{T-1}$ induced by one-period risk measure (1) through modified translation property (6) is recursively defined as

$\rho_{t, T}=w_{t}\left(X_{t}\right)+\rho_{t}\left(\rho_{t+1, T}\left(X_{t+1}, \ldots, X_{T}\right)\right), t=1, \ldots, T-1$

As aforementioned, time consistency is the most important issue of dynamic risk measures. One of the most commonly used versions is introduced in [12].

Definition 2. A dynamic risk measure $\left\{\rho_{t, T}\right\}_{t=1}^{T-1}$ is time consistent if for all $1 \leq \tau \leq \theta<T$ and all sequences $\left\{X_{t}\right\}_{t=1}^{T}$, $\left\{Y_{t}\right\}_{t=1}^{T} \in \mathrm{L}_{t, T}$,

$$
\begin{aligned}
& X_{k}=Y_{k}, k=\tau, \ldots, \theta-1 \text { and } \\
& \rho_{\theta, T}\left(X_{\theta}, \ldots, X_{T}\right) \leq \rho_{\theta, T}\left(Y_{\theta}, \ldots, Y_{T}\right)
\end{aligned}
$$


imply $\rho_{\tau, T}\left(X_{t}, \ldots, X_{T}\right) \leq \rho_{\tau, T}\left(Y_{t}, \ldots, Y_{T}\right)$.

This definition is intuitive since it indicates that if the future subsequence of sequence $X_{t}$ is at least as good as the subsequence of another sequence $Y_{t}$ and today's value of $X_{t}$ is the same as that of $Y_{t}$, then $X_{t}$ is at least as good as $Y_{t}$ from the perspective of today. We show that our new risk measure satisfies time consistency.

Proposition 6. Suppose a dynamic risk measure $\left\{\rho_{t, T}\right\}_{t=1}^{T-1}$ satisfies condition (6)-(7), then it is time consistent if and only if for all $1 \leq \tau \leq \theta<T$ and all $\left\{X_{t}\right\} \in \mathrm{L}_{1, T}$,

$$
\begin{aligned}
& \rho_{\tau, T}\left(X_{\tau}, \ldots, X_{\theta}, \ldots, X_{T}\right)= \\
& \quad \rho_{\tau, T}\left(X_{\tau}, \ldots, X_{\theta-1}, w_{\theta}^{-1} \circ \rho_{\theta, T}\left(X_{\theta}, \ldots, X_{T}\right), 0, \ldots, 0\right.
\end{aligned}
$$

where $w_{\tau}(\cdot)$ satisfies properties stated in Proposition 2 .

Proof. Suppose sequences $\left\{X_{t}\right\}_{t=1}^{T}, \quad\left\{Y_{t}\right\}_{t=1}^{T} \in \mathrm{L}_{t, T}$ satisfy the Equation (9), by the monotonicity of $\left\{\rho_{t, T}\right\}_{t=1}^{T-1}$ and $w_{\theta}$, it follows that

$$
\begin{aligned}
& \rho_{\tau, T}\left(X_{\tau}, \ldots, X_{\theta-1}, \rho_{\theta, T}\left(X_{\theta}, \ldots, X_{T}\right), 0, \ldots, 0\right. \\
& \leq \rho_{\tau, T}\left(Y_{\tau}, \ldots, Y_{\theta-1}, \rho_{\theta, T}\left(Y_{\theta}, \ldots, Y_{T}\right), 0, \ldots, 0\right)
\end{aligned} .
$$

If identity (10) holds, then

$$
\rho_{\tau, T}\left(X_{\tau}, \ldots, X_{T}\right) \leq \rho_{\tau, T}\left(Y_{\tau}, \ldots, Y_{T}\right) .
$$

\section{Portfolio Selection Model and Optimal Condition}

Based on the new dynamic risk measure, we establish a multistage portfolio selection model in this section. Suppose we have initial capital $X_{1}$ in $n$ assets at stage 1 , each of which has respective net expected return rate $r_{t}=\left(r_{1 t}, \ldots, r_{n t}\right)$ at stage $t=2, \ldots, T$, forming a random process with a known distribution (for example, can be determined by Vector Auto Regression model $\operatorname{VAR}(p))$. We assume this process is stagewise independent, i.e., $r_{t}$ is independent of $r_{1}, \ldots, r_{t-1}$ for $t=2, \ldots, T$. This assumption is not very realistic but in most cases, we can transform the across stage dependent process into stagewise independent by adding state variables to the model (cf. [13]). Suppose further a self-balance model, that is, we reallocate our portfolio at each stage $t=1, \ldots, T-1$, but without investing additional money during the time period. At each stage $t$, we decide the amount of the $n$ assets $x_{t}=\left(x_{1 t}, \ldots, x_{n t}\right)$, satisfying the balance of wealth constraints $\sum_{i=1}^{n} x_{i t}=\sum_{i=1}^{n}\left(1+r_{i t}\right) x_{i, t-1}, t=1, \ldots, T-1$. The sequence of decisions $x_{t}$ satisfies nonanticipativity (implementable) constraints, i.e., $x_{t}$ is a function of information available at the current stage, say the process $\left(r_{1}, \ldots, r_{t}\right)$. We assume there are no short sales or borrowing: $x_{i t} \geq 0$ for all $i$ and $t$. Our goal is minimizing the risk of the whole process over all the implementable and feasible policies, measured by our new dynamic risk measure $\rho_{1, T}\left(X_{1}, \ldots, X_{T}\right)$.

We write the dynamic programming equations for the multistage problem. At the stage $t=T-1, \ldots, 1$, a realization of $r_{[t]}:=\left(r_{1}, \ldots, r_{t}\right)$ is known. We solve the problem

$$
\begin{aligned}
& V_{t}\left(x_{t-1}\right):=\min _{x_{t} \geq 0} w_{t}\left(\sum_{i=1}^{n} x_{i t}\right)+\mathrm{V}_{t+1}\left(x_{t}\right) \\
& \text { s.t. } \sum_{i=1}^{n} x_{i, t}=\sum_{i=1}^{n}\left(1+r_{i, t}\right) x_{i, t-1}
\end{aligned}
$$

where

$\mathrm{V}_{t+1}\left(x_{t}\right):=\rho_{t}\left[V_{t+1}\left(x_{t}\right)\right], \mathrm{V}_{T}\left(x_{T-1}\right)=\rho_{T-1}\left(\sum_{i=1}^{n}\left(1+r_{i T}\right) x_{T-1}\right)$

By conjugate duality theory (c.f. [9]), we can prove the following optimal condition of problem (11).

Proposition 7. $x_{t}^{*}$ is an optimal solution of (11) if and only if there exists $\pi_{t}^{*} \in \mathrm{D}\left(x_{t-1}\right)$ such that $0 \in \partial L_{t}\left(x_{t}^{*}, \pi_{t}^{*}\right)$, where $\mathrm{D}\left(x_{t-1}\right)$ is the set of optimal solutions of the dual problem

$$
\begin{aligned}
& \max _{\pi_{t}}\left\{\pi_{t} \sum_{i=1}^{n}\left(1+r_{i t}\right) x_{i t}-\sup _{x_{t}}\left[\pi_{t} \sum_{i=1}^{n} x_{i t}-w_{t}\left(\sum_{i=1}^{n} x_{i t}\right)-\mathrm{V}_{t+1}\left(x_{t}\right)\right]\right\}, \\
& \text { and } \quad L_{t}\left(x_{t}, \pi_{t}\right) \quad \text { is } \quad \text { the e Lagrangian } \\
& L_{t}\left(x_{t}, \pi_{t}\right)=w_{t}\left(\sum_{i=1}^{n} x_{i t}\right)+\mathrm{V}_{t+1}\left(x_{t}\right)+\pi_{t}\left(\sum_{i=1}^{n}\left(1+r_{i t}\right) x_{i, t-1}-\sum_{i=1}^{n} x_{t}\right), \mathrm{a}
\end{aligned}
$$
nd the subdifferential of $f$ at $x$ is denoted as $\partial f(x)$. Further, the function $V_{t}$ is differentiable at $x_{t-1}$ and $\partial V_{t}\left(x_{t-1}\right)=\mathrm{D}_{t}\left(x_{t-1}\right) \mathbf{1 1}$, where $\mathbf{1 1}$ is a $n$ dimensional vector whose elements are 1 .

Proof. First, the function $V_{t}$ is convex for $t=1, \ldots, T$. Indeed, since $w_{T}$ and $\rho_{T}$ are convex and increasing, the convexity of $V_{T}$ follows the fact that the composition of increasing convex function is still convex and that the minimum function preserves convexity. By induction and increasing and convex property of $\rho_{t}$, convexity of $V_{t}, t=T-1, \ldots, 1$ is obtained. Next, since $V_{t}$ is finite and continuous with respect to $x_{t-1}$, by conjugate duality theory (c.f. Theorem 7.8 in [9]), we obtain the result.

The optimal condition and the subdifferential of $V_{t}$ is very useful practically. For example, it has been pointed 
out in [13] that the complexity of Sample Average Approximation (SAA) method for solving multistage stochastic programming grows exponentially in the number of scenarios and stages. An tractable way to solve the SAA problem approximately is by stochastic dual dynamic programming (SDDP) method. The subdifferential of $V_{t}$ is critical when deciding the SDDP algorithm. Indeed, we can modify the SDDP algorithm easily in [13] based on $\partial V_{t}$.

\section{Conclusion}

By linearly combining the downside measure and dispersion measure which punishes downside risk and rewards upside potential together, meanwhile distorting the financial value, this paper proposes a new class of one-period risk measure. The new static measure satisfies convexity, cash-subadditivity, preserves second order stochastic dominance, and can reflect investor's risk attitude. Based on this static measure, we then construct a dynamic time consistent risk measure using a modified translation property. Under this new dynamic measure, we establish a portfolio selection model whose goal is to minimize the risk of the whole process. By conjugate duality theory, we derive its optimal condition, which facilitates us implement the SDDP algorithm for solving the multistage stochastic program. We only consider several theoretical property of our new risk measure, nevertheless, whether such measure is practically ideal needs some empirical research using realistic data. This issue is left for future research.

\section{Acknowledgements}

I am grateful to Professor Alexander Shapiro in Georgia Institute of Technology for his sincere help on my work.

\section{REFERENCES}

[1] Konno, Hiroshi, and Hiroaki Yamazaki. "Mean-absolute deviation portfolio optimization model and its applications to Tokyo stock market." Management science 37, no. 5 (1991): 519-531. doi:10.1287/mnsc.37.5.519

[2] Bawa, Vijay S., and Eric B. Lindenberg. "Capital market equilibrium in a mean-lower partial moment framework."
Journal of Financial Economics 5, no. 2 (1977): 189-200. doi:10.1016/0304-405X(77)90017-4

[3] Artzner, Philippe, Freddy Delbaen, Jean - Marc Eber, and David Heath. "Coherent measures of risk." Mathematical finance 9, no. 3 (1999): 203-228. doi:10.1111/1467-9965.00068

[4] Föllmer, Hans, and Alexander Schied. "Convex measures of risk and trading constraints." Finance and Stochastics 6, no. 4 (2002): 429-447. doi:10.1007/s007800200072

[5] El Karoui, Nicole, and Claudia Ravanelli. "Cash subadditive risk measures and interest rate ambiguity." Mathematical Finance 19, no. 4 (2009): 561-590. doi:10.1111/j.1467-9965.2009.00380.x

[6] Rachev, Svetlozar, Sergio Ortobelli, Stoyan Stoyanov, J. FABOZZI FRANK, and Almira Biglova. "Desirable properties of an ideal risk measure in portfolio theory." International Journal of Theoretical and Applied Finance 11, no. 01 (2008): 19-54. doi:10.1142/S0219024908004713

[7] Cheridito, Patrick, Freddy Delbaen, and Michael Kupper. "Dynamic monetary risk measures for bounded discrete-time processes." Electronic Journal of Probability 11, no. 3 (2006): 57-106. doi:10.1214/EJP.v11-302

[8] Chen Zhiping, Li Yang, Daobao Xu, and Qianhui Hu. "Tail nonlinearly transformed risk measure and its application." OR Spectrum (2011): 1-44. doi:10.1007/s00291-011-0271-2

[9] Shapiro A., Dentcheva D., Ruszczynski A., Lectures on stochastic programming: modeling and theory. Society for Industrial Mathematics, Vol.9, 2009. doi: $10.1137 / 1.9780898718751$

[10] Denneberg, Dieter. "Premium Calculation." Astin Bulletin 20, no. 2 (1990): 181-190.doi:10.2143/AST.20.2.2005441

[11] Rockafellar, R. Tyrrell, and Stanislav Uryasev. "Optimization of conditional value-at-risk." Journal of risk 2 (2000): 21-42.

[12] Ruszczyński, Andrzej. "Risk-averse dynamic programming for Markov decision processes." Mathematical programming 125, no. 2 (2010): 235-261. doi:10.1007/s10107-010-0393-3

[13] Alexander Shapiro, Wajdi Tekaya, Joari Paulo da Costa, Murilo Pereira Soares, "Risk neutral and risk averse Stochastic Dual Dynamic Programming method", European Journal of Operations Research, vol. 224, pp. 375-391, 2013. doi:10.1016/i.ejor.2012.08.022 